\title{
APPLICATION OF CHIRAL NUCLEAR FORCES TO LIGHT NUCLEI
}

\author{
A. NOGGA* \\ Institut für Kernphysik, Forschungszentrum Jülich, 52425 Jülich, Germany \\ *E-mail: a.nogga@fz-juelich.de
}

\begin{abstract}
In these proceedings, we discuss the current status of nuclear bound state predictions based on chiral nuclear interactions. Results of ordinary $s$ - and $p$-shell nuclei and light hypernuclei are shown.
\end{abstract}

Keywords: chiral nuclear interaction, nuclear binding energies, hypernuclei

\section{Introduction}

One main goal of nuclear physics is the understanding of the binding energies of nuclei. In the past, it was the aim to relate the binding energies to nuclear forces that describe the two-nucleon (NN) scattering data. This, however, can only be part of a complete understanding. Finally, it is necessary to related the binding energies to QCD.

The most promising approach to establish this relation is chiral effective field theory. It enables us to build into the nuclear interaction the symmetries of QCD and allows the determination of unknown parameters by adjustment of lattice calculations even at unphysically large quark masses. In this way, a direct connection of nuclear binding energies and QCD will be established in the future. ${ }^{1}$

At this time, chiral effective theory is an important guideline to identify the most important contributions to nuclear interactions and to pin down relations of nuclear interactions to other strong interaction processes, e.g. $\pi \mathrm{N}$ scattering and $\pi$ production. In this context, it is of utmost importance to pin down the structure of three-nucleon forces (3NF's). Traditional calculations ${ }^{2-4}$ clearly show that 3 NF's are significant for a quantitative description of binding energies. Current models can provide correct binding for $s$-shell, ${ }^{2}$ but fail for $p$-shell nuclei ${ }^{5}$ and scattering observables ${ }^{6-8}$ (see also contributions of Johan Messchendorp and Kimiko Sekiguchi to this 
conference $\left.{ }^{9,10}\right)$. Extensions of these models can improve some of these failures $^{5}$ in the regime of light nuclei, but again some deviations show up for the more complex systems. ${ }^{11}$ A reliable extension, however, is the key to get theoretical insight into the structure of, e.g., exotic nuclei.

Therefore, the application of chiral perturbation theory to the nuclear bound state problem is of interest to make nuclear structure calculations more reliable. We will argue below, that such calculations are also important to confirm that we correctly extend chiral perturbation theory to the nonperturbative nuclear systems.

In these proceedings, we discuss the current status of bound state calculations for light nuclei and hypernuclei. In Section 2, we define the chiral interactions used for the calculations. We then look in detail at the dependence of the results on the cutoff necessary for the regularization of the problem in Section 3. Section 4 is devoted to predictions for $p$-shell nuclei. Then, we turn to first results for hypernuclei in Section 5 and conclude in Section 6 .

\section{Chiral nuclear forces}

For a complete overview, we refer to the recent reviews on chiral nuclear interactions $^{12,13}$ (see also Evgeny Epelbaum's contribution to this conference $\left.^{14}\right)$. Here we will only give a summary of the main results important for the further discussion.

A direct application of the power counting of chiral perturbation theory to nuclear systems is not possible. The existence of nuclear bound states excludes any non-perturbative approach. Weinberg realized that this non-perturbative behavior is caused by an enhancement of diagrams with purely nucleonic intermediate states. ${ }^{15} \mathrm{He}$ classified such diagrams as "reducible" and conjectured that the power counting of chiral perturbation theory can be applied to the "irreducible" diagrams. These diagrams then need to be summed to all orders using a numerical technique, e.g. solving the Lippmann-Schwinger equation. In this way, one obtains in a systematic way a nuclear interaction based on a chiral Lagrangian. The interaction kernel is expanded in powers of a typical momentum in nuclei or the $\pi$ mass (which is a generic small scale $Q$ ) over the chiral symmetry breaking scale $\Lambda_{\chi} \approx 1 \mathrm{GeV}$. The power counting justifies straightforwardly the common assumption that NN interactions are much more important than 3NF's. Higher order forces are even further suppressed. Quantitative results ${ }^{16-19}$ confirmed the approach for the NN system.

The more complex few-nucleon systems, however, promise further chal- 
lenges for this approach. Since 3NF's become quantitatively important, the few-nucleon observables are sensitive to subleading parts of the nuclear interactions. Especially, the binding energies are sensitive to details of the interaction, since they are the result of a rather large cancelation of kinetic energy and potential energy.

In order $Q^{3}$ first 3 NF's appear. ${ }^{20}$ Three topologies exist. Chiral symmetry relates the strength of the the $2 \pi$ exchange $3 \mathrm{NF}$ to corresponding diagrams of the $\mathrm{NN}$ interaction and also to $\pi \mathrm{N}$ scattering. A quantitative confirmation that consistent values of the corresponding low energy constants (LEC's) (usually referred to as $c_{i}$ ) can be found for all of these processes is an important confirmation that chiral symmetry is realized in nuclear interactions in the way we assume now. At this point fairly consistent values have been extracted from $\mathrm{NN}$ and $\pi \mathrm{N}$ data. ${ }^{21-25}$ For a more conclusive comparison, the extractions have to be more accurate or additional insight from few-nucleon systems is required.

Except for the $2 \pi$ exchange $3 \mathrm{NF}$ 's, chiral effective theory predicts two more leading $3 \mathrm{NF}$ structures. Here two a priori unknown LEC's appear. The first one determines the strength of the $1 \pi$ exchange diagram and can in principle be related to $\pi$ production in NN scattering ${ }^{26}$ or weak processes in few-nucleon systems. ${ }^{27}$ In practice, such extractions cannot be used at this time, since they were performed in frame works that are not consistent with the one used here. The second one enters via the $6 \mathrm{~N}$ contact vertex. It can only be fixed by matching to few-nucleon observables.

\section{Cutoff dependence of nuclear binding energies}

In any order, the chiral potentials are singular interactions. If the singularities are attractive, the Hamiltonian becomes unbounded from below. Therefore, regularization is required before solving the Lippmann-Schwinger or Schrödinger equation based on chiral interactions. Usually the regularization is performed by means of a momentum cutoff $\Lambda$. The available realizations of chiral interactions mostly use $\Lambda \approx 500-600 \mathrm{MeV}$. Here $\Lambda$ is chosen to be below a typical hadron mass, e.g. the $\rho$ mass. A quantitative confirmation for this choice is desirable. To this aim, it is instructive to study the cutoff dependence in a much larger range of $\Lambda$ 's. For a few-body problem this was first done $\mathrm{in}^{28}$ for the leading order nuclear interaction only. It turned out that in leading order, additional contact interactions beyond those required by naive dimensional analysis are required to describe the NN data cutoff independently. ${ }^{28-30}$ These results triggered an ongoing discussion on the proper power counting for chiral nuclear interactions (see 
Table 1. Expected variations of the results with the cutoff for different orders of the interaction. $n$ is the order of the missing contact interactions, $\Lambda$ the momentum cutoff used and $\Delta V / V(\Delta E / E)$ are the estimated relative variations of the potential (binding) energy.

\begin{tabular}{ccclr} 
order & $n$ & $\Lambda[\mathrm{MeV}]$ & $\Delta V / V$ & $\Delta E / E$ \\
\hline$Q^{0}$ & 2 & 500 & $7 \%$ & $70 \%$ \\
$Q^{2}$ & 4 & 500 & $0.5 \%$ & $5 \%$ \\
$Q^{3}$ & 4 & 500 & $0.5 \%$ & $5 \%$ \\
$Q^{4}$ & 6 & 500 & $0.03 \%$ & $0.3 \%$ \\
\hline$Q^{0}$ & 4 & 700 & $0.1 \%$ & $1 \%$
\end{tabular}

e.g. the panel discussion in the few-body working group $\left.{ }^{31-33}\right)$. Whereas this issue will be of importance to extend the nuclear interaction to momenta close to the $\pi$ production threshold, ${ }^{34}$ where the currently used cutoffs become similar to typical momenta, we will argue below that it does not strongly affect progress for nuclear structure calculations. The differences discussed in the power counting become quantitatively negligible for high order interactions and for cutoffs of the order of $500 \mathrm{MeV}$.

It is useful to discuss first our expectations for the cutoff dependence of chiral interactions. To get a rough estimate, one can assume that the typical small scale (typical momentum or pion mass) is of the order $Q \approx 130 \mathrm{MeV}$. One can expect that variations of the result are absorbed by contact interactions that are not considered at a given order. Assuming natural size, these contact interactions should give contributions that scale with $(Q / \Lambda)^{n}$. Given that NN contact interactions contribute only in even orders, one finds that $n=2$ in leading order $\left(Q^{0}\right), n=4$ in next-leading order $\left(\mathrm{NLO}, Q^{2}\right)$ and next-to-next-to-leading order (N2LO, $\left.Q^{3}\right)$ and $n=6$ for order $Q^{4}(\mathrm{~N} 3 \mathrm{LO})$ interactions. $\Lambda \approx 500 \mathrm{MeV}$ for typical realizations of chiral interactions. Table 1 shows the expected variation of the potential energy for $\Lambda=500$ and $700 \mathrm{MeV}$. Assuming that these missing contributions can be treated in perturbation theory, one finds that this is the actual change expected for the binding energy. This, however, implies that the relative variation of the binding energy becomes quite large. In the table, we simple assumed that it is one order of magnitude larger than the relative variation of the potential. The table also shows that the actual size of the cutoff variation is similar for NLO and N2LO (at least if only NN interactions are considered) and that the estimate strongly depends on the cutoff, especially for higher order interactions. Only in order $Q^{4}$, the variation of the binding energy with the cutoff is expected to be very small. It is now interesting to confront these expectations with actual calculations.

Fig. 1 shows the dependence of the ${ }^{3} \mathrm{H}$ binding energy on $\Lambda$ for a wide 


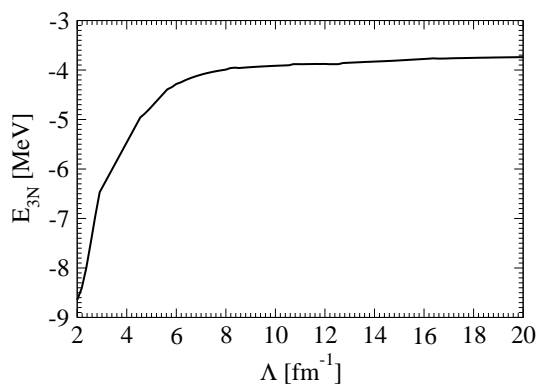

Fig. 1. Dependence of the ${ }^{3} \mathrm{H}$ binding energy on the cutoff $\Lambda$ for the leading order interaction. Thereby additional contact interactions were required. ${ }^{28}$

Table 2. Calculated dependence of the ${ }^{3} \mathrm{H}$ binding energy for different chiral interactions. The $Q^{0}, Q^{2}, Q^{3}$ and $Q^{4}$ interactions are from Refs. ${ }^{18,19,28} \Lambda$ is the momentum cutoff imposed on the Lippmann-Schwinger equation, $\tilde{\Lambda}$ are cutoffs imposed on internal loops (see Ref. ${ }^{19}$ ). DR notes that loops are dimensionally regulated.

\begin{tabular}{cc|ccc|c} 
order & $\Lambda / \tilde{\Lambda}[\mathrm{MeV}]$ & $E[\mathrm{MeV}]$ & $V[\mathrm{MeV}]$ & $\Delta E[\mathrm{keV}]$ & $\Delta E / V$ \\
\hline$Q^{0}$ & $500 /$ no loops & -7.50 & -51.8 & 1430 & $3.0 \%$ \\
& $600 /$ no loops & -6.07 & & & \\
\hline$Q^{2}$ & $400 / 700$ & -8.46 & & 650 & $1.6 \%$ \\
& $550 / 700$ & -7.81 & -41.1 & & \\
\hline$Q^{3}$ & $450 / 700$ & -8.42 & -38.3 & 530 & $1.3 \%$ \\
& $600 / 700$ & -7.89 & & & \\
\hline$Q^{4}$ & $500 / \mathrm{DR}$ & -7.84 & -42.3 & 40 & $0.1 \%$ \\
& $600 / \mathrm{DR}$ & -7.80 & & &
\end{tabular}

range of cutoffs between $\Lambda=2-20 \mathrm{fm}^{-1}(\approx 400-5000 \mathrm{MeV})$. One observes that one can obtain a cutoff independent binding energy for large $\Lambda$, once the cutoff dependence of $\mathrm{NN}$ predictions is removed by promoting counter terms from naively higher orders. Contrarily to the effective theory without pions, ${ }^{35}$ one does not need to promote a $3 \mathrm{NF}$ to leading order to get cutoff independent results. In the range of cutoffs considered, one obtains a variation of the result of approximately $4 \mathrm{MeV}$ consistent with the expected variation of a leading order calculation. Note also that in the low cutoff range between 500 and $600 \mathrm{MeV}$, one finds that the binding energy varies quite rapidly by approximately $1.5 \mathrm{MeV}$.

In view of the fact that so far no higher order realization of chiral interactions has been developed that covers a similarly large range of cutoffs, it is not possible to study the binding energies in a similar manner for the higher orders. An order of magnitude estimate, however, is possible by com- 
Table 3. Strength constants of the $1 \pi$ exchange and $6 \mathrm{~N}$ contact $3 \mathrm{NF}$ 's (see definition in Ref. ${ }^{36}$ ). $Q^{3}$ interactions are from Ref. ${ }^{19} Q^{4}$ interaction is from Ref. ${ }^{18} 3 \mathrm{NF}-\mathrm{A}$ and $3 \mathrm{NF}-\mathrm{B}$ label two sets of parameters that describe the $3 \mathrm{~N}$ and $4 \mathrm{~N}$ binding energies equally well.

\begin{tabular}{l|lrr} 
interaction & $\Lambda / \tilde{\Lambda}[\mathrm{MeV}]$ & $c_{D}$ & $c_{E}$ \\
\hline$Q^{3}$ & $450 / 700$ & 1.20 & -0.082 \\
$Q^{3}$ & $600 / 700$ & -4.27 & -1.25 \\
$Q^{4}-3 \mathrm{NF}-\mathrm{A}$ & $500 / \mathrm{DR}$ & -1.11 & -0.66 \\
$Q^{4}-3 \mathrm{NF}-\mathrm{B}$ & $500 / \mathrm{DR}$ & 8.14 & -2.02
\end{tabular}

paring the variation within a small range of $\Lambda \approx 500-600 \mathrm{MeV}$. Neglecting the contribution of 3NF's, for which we assume a rather cutoff independent contribution to the binding energies, this is shown in Table 2 again for ${ }^{3} \mathrm{H}$ for several orders of the chiral expansion. Though the variations within the range of cutoffs are somewhat large compared to the estimate in Table 1, the results still confirm the power counting expectation. Quantitatively, the cutoff dependence becomes negligible in order $Q^{4}$ (N3LO).

For a quantitative calculation in higher orders, we need to fix the LEC's of the 3NF. As discussed above, there are two LEC's unrelated to the NN interaction in the leading 3NF's. Therefore, one needs two few-body data. Most naturally, we use the ${ }^{3} \mathrm{H}$ binding energy in all of your determinations of these LEC's. Both, the ${ }^{4} \mathrm{He}$ binding energy and the doublet neutrondeuteron scattering length are suitable to constrain the second LEC. The details of the determinations can be found in Refs. ${ }^{36,37}$ We have performed the fits for combinations of the leading $3 \mathrm{NF}$ with the $Q^{3}$ chiral interactions of Ref. ${ }^{19}$ and the $Q^{4}$ interaction of Ref. ${ }^{18}$ The latter combination is not strictly consistent with the power counting, since we neglected $Q^{4}$ contributions to the $3 \mathrm{NF}$ and the $4 \mathrm{NF} .{ }^{38}$ The values of the parameters $c_{D}$ and $c_{E}$ in the notation of Ref. ${ }^{36}$ are given in Table 3 for completeness. Note that we find two solutions for the LEC's in conjunction with the NN force of Ref., ${ }^{18}$ which we are labeled 3NF-A and 3NF-B in the following.

The binding energies of ${ }^{3} \mathrm{H}$ and ${ }^{4} \mathrm{He}$ are well described by the chiral interactions (by construction). To confirm that the application of chiral interactions to $s$-shell nuclei gives consistent results, it is interesting to compare the contribution of 3NF's to the ${ }^{3} \mathrm{H}$ and ${ }^{4} \mathrm{He}$ potential energies to a power counting estimate. The $3 \mathrm{NF}$ is formally in order $Q^{3}$. An estimate similar to the one shown in Table 1 leads to an expected contribution of the $3 \mathrm{NF}$ of $\approx 2 \%$ to the total potential energy. Our calculations show that the $3 \mathrm{NF}$ (even the various parts of it) do not contribute more than $7.5 \%$ to the potential energy of ${ }^{4} \mathrm{He}$, which is still in line with the power counting estimate. Therefore, we note that the bound state calculations for 

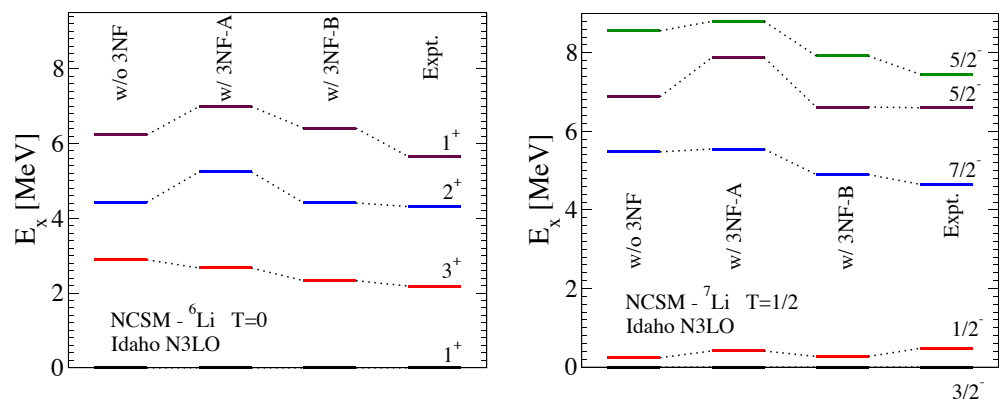

Fig. 2. Spectra of ${ }^{6} \mathrm{Li}$ and ${ }^{7} \mathrm{Li}$ nuclei based on the chiral interaction of Ref. ${ }^{18}$ without $3 \mathrm{NF}$ or in combination with $3 \mathrm{NF}-\mathrm{A}$ and $3 \mathrm{NF}-\mathrm{B}$. See text for definitions.

the $s$-shell nuclei confirm our expectations from the power counting.

\section{Predictions for ${ }^{6} \mathrm{Li}$ and ${ }^{7} \mathrm{Li}$}

With the 3NF's completely fixed, we are now in the position to make predictions for $p$-shell nuclei. All results for the $p$-shell nuclei have been obtained for the $Q^{4}$ interaction of Ref. ${ }^{18}$ Since the cutoff dependence for an a $Q^{4}$ chiral interaction is negligible small, we will therefore restrict ourselves only to one cutoff $\Lambda=500 \mathrm{MeV}$.

To predict binding energies and spectra of $p$-shell nuclei, we need to use a technique for solving the Schrödinger equation based on non-local interactions. Here, we will show results based on the "no-core shell model" approach (NCSM). Details of the technique and the results for ${ }^{7} \mathrm{Li}$ are discussed in. ${ }^{39}$ Here, it is sufficient to emphasize that excitation energies can be accurately obtained by the NCSM. The accuracy of the binding energy can be estimate to be approximately $1 \mathrm{MeV}$.

Since the NCSM results for the spectra are more accurate than the binding energies, they are especially important to study the chiral interactions. As can be seen in Fig. 2, the excitation energies are changed by the addition of the 3NF. Note that the two parameter sets 3NF-A and 3NF-B, that describe the $s$-shell nuclei equally well, result in different predictions for ${ }^{6} \mathrm{Li}$ and ${ }^{7} \mathrm{Li}$. The expected sensitivity to the structure of the $3 \mathrm{NF}$ is confirmed by these calculations. For ${ }^{6} \mathrm{Li}$ and ${ }^{7} \mathrm{Li}$, we find a consistently better description of the spectra for parameter set $3 \mathrm{NF}-\mathrm{B}$ compared to the predictions of $3 \mathrm{NF}-\mathrm{A}$ and without $3 \mathrm{NF}$.

For the binding energies, the situation is somewhat different. Our results are compiled in Table 4. Again 3NF-B improves the description of the radii, 
Table 4. Binding energies $E$ and point proton radii $r_{p}$ for ${ }^{6} \mathrm{Li}$ and ${ }^{7} \mathrm{Li}$. Results for chiral interactions are compared to results based on phenomenological interactions ${ }^{5}$ and to experimental values that a corrected for the finite size of the protons.

\begin{tabular}{l|cccc}
\multirow{2}{*}{ interaction } & \multicolumn{2}{|c}{${ }^{6} \mathrm{Li}$} & \multicolumn{2}{c}{${ }^{6} \mathrm{Li}$} \\
& $E[\mathrm{MeV}]$ & $r_{p}[\mathrm{fm}]$ & $E[\mathrm{MeV}]$ & $r_{p}[\mathrm{fm}]$ \\
\hline$Q^{4}-$ no 3NF & -30.0 & 2.20 & -34.6 & 2.15 \\
$Q^{4}-$ 3NF-A & -32.3 & 2.16 & -38.0 & 2.11 \\
$Q^{4}-3$ NF-B & -31.1 & 2.25 & -36.7 & 2.23 \\
\hline AV18 - IL2 & -32.3 & 2.39 & -38.9 & 2.25 \\
AV18 - Urb-IX & -31.1 & 2.57 & -37.5 & 2.33 \\
\hline Expt. & -32.0 & 2.43 & -39.2 & 2.27
\end{tabular}

but both $p$-shell nuclei appear to be underbound.

This apparent inconsistency of the results deserves some further consideration. Here, it is important to note that we have fixed the strength of the $2 \pi$ exchange part of the $3 \mathrm{NF}$ using the choice of $c_{i}$ of the NN potential of Ref. ${ }^{18}$ The description of the NN data is not very sensitive to the choice of these parameters. Nuclear matter calculations for low momentum interactions including the same 3NF's, however, indicate that a change of the $c_{i}$ 's by only $10 \%$, correcting the $c_{D}$ and $c_{E}$ so that the $s$-shell nuclei do not change their binding energy, may change the binding energy per particle in symmetric nuclear matter by $1 \mathrm{MeV} \cdot{ }^{40,41}$ It is therefore conceivable that a consistent description of binding energies and spectra can be obtained by a variation of the $c_{i}$ 's. This needs to be explicitly checked in the future.

Finally, we note that the addition of 3NF-B, though the relatively low cutoffs remove any strong short range repulsion, increases both the binding energy and the radii.

\section{Hypernuclei}

Now we turn to hypernuclear binding energies. Recently, Polinder and collaborators have developed a first realization of the chiral hyperon-nucleon (YN) interaction. ${ }^{42}$ A systematic approach to the problem of the YN interaction is badly needed. It will enable us to understand the way flavor SU(3) symmetry is broken in nuclear systems. Also the impact of hyperons on the nuclear equation of state is possibly significant also for astrophysical applications ${ }^{43}$ but the pour knowledge of the interactions of hyperons hinders more insight. Most of these issues are due to the very scarce set of data in the YN sector. Moreover, most of the data are considerably above the $\Lambda \mathrm{N}$ threshold. Therefore, even the scattering lengths for $\Lambda \mathrm{N}$ scattering are essentially unknown (for a discussion on the current status see e.g. ${ }^{44,45}$ ). 
Table 5. $\Lambda$ separation energies of the $0^{+}\left(E_{\text {sep }}\left(0^{+}\right)\right)$and $1^{+}\left(E_{\text {sep }}\left(1^{+}\right)\right)$states and their difference $\Delta E_{\text {sep }}$ for ${ }^{4} \mathrm{H}$ and the difference of the separation energies for the mirror hypernuclei ${ }_{\Lambda}^{4} \mathrm{He}$ and ${ }_{\Lambda}^{4} \mathrm{H}\left(\mathrm{CSB}-0^{+}\right.$and $\left.\mathrm{CSB}-1^{+}\right)$. Results for the chiral YN interaction for various cutoffs $\Lambda$ are compared to results for two phenomenological models ${ }^{46,47}$ and the experimental values.

\begin{tabular}{|c|c|c|c|c|c|c|c|}
\hline$\Lambda[\mathrm{MeV}]$ & 500 & 550 & 650 & 700 & Jülich 05 & Nijm SC97f & Expt. \\
\hline$E_{\text {sep }}\left(0^{+}\right)[\mathrm{MeV}]$ & 2.63 & 2.46 & 2.36 & 2.38 & 1.87 & 1.60 & 2.04 \\
\hline$E_{\text {sep }}\left(1^{+}\right)[\mathrm{MeV}]$ & 1.85 & 1.51 & 1.23 & 1.04 & 2.34 & 0.54 & 1.00 \\
\hline$\Delta E_{\text {sep }}[\mathrm{MeV}]$ & 0.78 & 0.95 & 1.13 & 1.34 & -0.48 & 0.99 & 1.04 \\
\hline${\mathrm{CSB}-0^{+}[\mathrm{MeV}]}$ & 0.01 & 0.02 & 0.02 & 0.03 & -0.01 & 0.10 & 0.35 \\
\hline $\mathrm{CSB}_{-1}{ }^{+}[\mathrm{MeV}]$ & -0.01 & -0.01 & -0.01 & -0.01 & — & -0.01 & 0.24 \\
\hline
\end{tabular}

Current models of the YN interaction ${ }^{46-48}$ do all describe the available YN data, but predictions for non-measured observables vary very strongly. They also fail to describe the measured binding energies of the light hypernuclei ${ }^{49}$ and, therefore, a more systematic insight into the YN interaction is even more badly needed.

Also the leading chiral interaction (one Goldstone-boson exchange and five non-derivative contact interactions) has been fitted to the scarce data base for the YN system. Additionally, the scattering lengths have been constrained, so that the ${ }_{\Lambda}^{3} \mathrm{H}$ binding energy is in agreement with the experimentally know value of $E=-2.35 \mathrm{MeV}$. Thereby, the cutoff was varied between 550 and $700 \mathrm{MeV}$ (for details see Ref. ${ }^{42}$ ). Contrarily to common expectation, the resulting $\Lambda \mathrm{N}$ cross section at low energies was much smaller than traditional models predict. In view of this surprisingly weak interaction, it is astonishing to find ${ }_{\Lambda}^{3} \mathrm{H}$ binding energies in agreement with experiment.

It is now important to confront predictions based on the leading order chiral interaction for the more complex hypernuclei ${ }_{\Lambda}^{4} \mathrm{H}$ and ${ }_{\Lambda}^{4} \mathrm{He}$ with the data. Two states, the $J^{\pi}=0^{+}$ground and a $J^{\pi}=1^{+}$exited state, of these mirror hypernuclei are experimentally known. Though the YN interactions are not very strongly constrained by the YN data, it has proven to be difficult to obtain a consistent description of both of the states and also of the well known charge dependence of the $\Lambda$ separation energies. It turned out that the splittings between the $0^{+}$and $1^{+}$state and the charge dependence of the separation energies are correlated with the strong $\Lambda-\Sigma$ conversion process. Table 5 compiles the new results based on the chiral interaction together with model predictions and the experimental values. The dependence on the NN interaction is mild. ${ }^{49}$ Here, we used the one of Ref. ${ }^{18}$ The leading order YN interaction results in very reasonable $\Lambda$ separation energies. The separation energy of the $0^{+}$state appears to be very cutoff independent, whereas the one of the $1^{+}$state is more strongly 
dependent on the cutoff in leading order. Given that the results are leading order only, they are very encouraging. NLO calculation will be important to confirm that the agreement improves and the cutoff dependence shrinks as we have seen for the ordinary nuclei. The leading order calculation does not include any charge symmetry breaking contributions in the interaction. As a result, the charge dependence of the separation energies is very small. (The small non-zero contributions is due to the $\Sigma^{+}-\Sigma^{-}$mass difference and the Coulomb interaction. Both has been included in the calculations of Table 5.) The NLO interaction will explicitly include first charge symmetry breaking contributions. Therefore, an improvement of the leading order results in this respect is also conceivable.

\section{Conclusions and Outlook}

We discussed the results for nuclear binding energies of ordinary $s$-shell and $p$-shell nuclei and the lightest hypernuclei based on chiral interactions. A special emphasis was on the confirmation of the power counting underlying nuclear interactions.

For the ordinary nuclei, the results of LO, NLO and N2LO chiral interactions confirm our power counting expectations. We find that N3LO $\mathrm{NN}$ interactions give predictions that are only insignificantly cutoff dependent. In this order, chiral interaction will become quantitatively useful for predictions of nuclear binding energies. In this context, the outcome of an on-going discussion on possible promotions of contact interactions for formerly higher order will be important, since it might enable us to extend the current realizations of chiral interactions to slightly larger values of the cutoffs closer to $\Lambda_{\chi}$ and, thereby, possibly improve the convergence of the chiral expansion.

The predictions for $p$-shell nuclei confirm the expected sensitivity of the 3NF's. Spectra and binding energies are improved by their addition. It will be interesting to allow for small variations of the pertinent LEC's $c_{i}$ to further improve the description, especially of the binding energies. In this way, an more accurate determination of the $c_{i}$ might become possible.

We also discussed first results for binding energies of the lightest hypernuclei and found that the separation energies of ${ }_{\Lambda}^{3} \mathrm{H}$ and ${ }_{\Lambda}^{4} \mathrm{H}$ can be consistently described. Now the extension to NLO will be very interesting. First, it should shrink the dependence on the cutoff and enable combined fits of the $\mathrm{NN}$ and $\mathrm{YN}$ interaction. Also first charge-symmetry breaking terms contribute to the YN interaction in this order. Since phenomenological models do not describe the charge-symmetry breaking of the ${ }_{\Lambda}^{4} \mathrm{H}-{ }_{\Lambda}^{4} \mathrm{He}$ 
properly, the results of the chiral interaction for this observable will be especially interesting.

\section{Acknowledgments}

I very much thank B. Barrett, E. Epelbaum, W. Glöckle, J. Golak and U. Meißner, P. Navrátil, H. Polinder, R. Skibiński, R. Timmermans, U. van Kolck, J. Vary, H. Witała for collaborating on the work presented here. The numerical calculations have in part been performed on the JUMP and JUBL computers of the NIC, Jülich, Germany.

\section{References}

1. M. Savage, Few-body lattice calculations, contribution to this conference.

2. A. Nogga, H. Kamada, W. Glöckle and B. R. Barrett, Phys. Rev. C 65, p. 054003(May 2002).

3. S. C. Pieper and R. B. Wiringa, Ann. Rev. Nuc. Part. Sci. 51, 53 (2001).

4. P. Navrátil and B. R. Barrett, Phys. Rev. C 57, 3119(June 1998).

5. S. C. Pieper, V. R. Pandharipande, R. B. Wiringa and J. Carlson, Phys. Rev. C 64, p. 014001(July 2001).

6. K. Sekiguchi et al., Phys. Rev. C 65, p. 034003(March 2002).

7. S. Kistryn et al., Phys. Rev. C 72, p. 044006(October 2005).

8. K. Ermisch et al., Phys. Rev. C 71, p. 064004(June 2005).

9. J. Messchendorp, Results from kvi: Nd elastic scattering, contribution to this conference.

10. K. Sekiguchi, Results on three-nucleon experiments from riken, contribution to this conference.

11. S. C. Pieper, Recent results from quantum monte carlo calculations of light nuclei, in Workshop on "Electron-Nucleus Scattering IX", (Isola d'Elba, Italy, 2006). http://conferences.jlab.org/elba/talks/pieper.pdf.

12. E. Epelbaum, Prog. Part. Nucl. Phys. 57, 654 (2006).

13. P. F. Bedaque and U. van Kolck, Ann. Rev. Nuc. Part. Sci. 52, 339 (2002).

14. E. Epelbaum, Towards a systematic theory of nuclear forces and nuclear currents, contribution to this conference.

15. S. Weinberg, Nucl. Phys. B 363, 3(September 1991).

16. C. Ordóñez, L. Ray and U. van Kolck, Phys. Rev. C 53, 2086(May 1996).

17. E. Epelbaum, W. Glöckle and U.-G. Meißner, Nucl. Phys. A 671, 295(May 2000).

18. D. R. Entem and R. Machleidt, Phys. Rev. C 68, p. 041001(October 2003).

19. E. Epelbaum, W. Glöckle and U.-G. Meißner, Nucl. Phys. A 747, 362(January 2005).

20. U. van Kolck, Phys. Rev. C 49, 2932(June 1994).

21. U.-G. Meißner, PoS LAT2005, p. 009 (2006).

22. M. C. M. Rentmeester, R. G. E. Timmermans and J. J. de Swart, Phys. Rev. $C$ 67, p. 044001(April 2003). 
23. D. R. Entem and R. Machleidt, Phys. Rev. C 66, p. 014002(July 2002).

24. P. Büttiker and U.-G. Meißner, Nucl. Phys. A 668, 97(March 2000).

25. N. Fettes, U.-G. Meißner and S. Steininger, Nucl. Phys. A 640, 199(September 1998).

26. C. Hanhart, U. van Kolck and G. A. Miller, Phys. Rev. Lett. 85, 2905(October 2000).

27. A. Gårdestig and D. R. Phillips, Phys. Rev. Lett. 96, p. 232301(June 2006).

28. A. Nogga, R. G. E. Timmermans and U. van Kolck, Phys. Rev. C 72, p. 054006(November 2005).

29. M. Pavón Valderrama and E. Ruiz Arriola (2005), nucl-th/0507075.

30. M. C. Birse, Phys. Rev. C 74, p. 014003(July 2006).

31. U. van Kolck, Panel discussion on power counting, contribution to this conference.

32. U.-G. Meißner, On the consistency of weinberg's power counting, contribution to this conference.

33. M. Pavón Valderrama, Renormalization of singular potentials and power counting, contribution to this conference.

34. A. Nogga, A. C. Fonseca, A. Gårdestig, C. Hanhart, C. J. Horowitz, G. A. Miller, J. A. Niskanen and U. van Kolck, Phys. Lett. B 639, 465(2006).

35. E. Braaten and H. W. Hammer, Phys. Rep. 428, 259(June 2006).

36. E. Epelbaum, A. Nogga, W. Glöckle, H. Kamada, U.-G. Meißner and H. Witała, Phys. Rev. C 66, p. 064001(December 2002).

37. A. Nogga, E. Epelbaum, P. Navrátil, W. Glöckle, H. Kamada, U.-G. Meißner, H. Witała, B. R. Barrett and J. P. Vary, Nucl. Phys. A 737, 236(June 2004).

38. E. Epelbaum, Phys. Lett. B 639, 456(August 2006).

39. A. Nogga, P. Navrátil, B. R. Barrett and J. P. Vary, Phys. Rev. C 73, p. 064002(June 2006).

40. A. Nogga, S. K. Bogner and A. Schwenk, Phys. Rev. C 70, p. 016002(December 2004).

41. S. K. Bogner, A. Schwenk, R. J. Furnstahl and A. Nogga, Nucl. Phys. A 763, 59(December 2005).

42. H. Polinder, J. Haidenbauer and U.-G. Meißner, Nucl. Phys. A779, 244 (2006).

43. B. D. Lackey, M. Nayyar and B. J. Owen, Phys. Rev. D 73, p. 024021(January 2006).

44. A. Gasparyan, J. Haidenbauer, C. Hanhart and J. Speth, Phys. Rev. C 69, p. 034006(March 2004).

45. A. Gasparyan, J. Haidenbauer and C. Hanhart, Phys. Rev. C 72, p. 034006(September 2005).

46. J. Haidenbauer and U.-G. Meißner, Phys. Rev. C 72, p. 044005(October 2005).

47. T. A. Rijken, V. G. J. Stoks and Y. Yamamoto, Phys. Rev. C 59, 21(January 1999).

48. T. A. Rijken and Y. Yamamoto, Phys. Rev. C 73, p. 044008(April 2006).

49. A. Nogga, H. Kamada and W. Glöckle, Phys. Rev. Lett. 88, p. 172501(April 2002). 